\title{
Introducing a New Tool for Studying Unstable Systems
}

\author{
František Gazdoš \\ Tomas Bata University in Zlín, Faculty of Applied Informatics, nam. T. G. Masaryka 5555, 76001 Zlin, Czech Republic
}

\begin{abstract}
This paper presents the starting project of a web site focussed on unstable systems. It is a web-based database in a bilingual version (English/Czech), which can be used as an information database for models of unstable processes. The web site contains the mathematical models of such systems, including their simulation files together with basic information about the stability of dynamic systems. The paper outlines the motivation for the development of this database, presents its basic structure, and discusses several models from the site. The areas of prospective usage are also suggested together with the possible directions of further development of this project. The contribution ends with a case study using the database for control system analysis and design of the Amira inverted pendulum. The systematic polynomial approach is fruitfully utilised for the task together with some useful tools from the robust control theory.
\end{abstract}

Keywords: Unstable systems, stability, modelling, simulation, database, robust control, polynomial approach.

\section{Introduction}

There are many processes in industrial practice with unstable behavior. These can be, for example, various types of reactors, distillation columns, combustion systems, etc ${ }^{[1,2]}$. There are also numerous systems in the environmental and social fields that are naturally unstable. Besides this, some systems in the military and aviation areas are deliberately designed to be unstable to gain better manoeuverability and to increase the speed of command responses. Consequently, all these systems are more difficult to control than the stable ones and are of special interest for designers and control engineers. They have to pay extra attention to implement safe control systems. If they fail, the consequences can be catastrophic $^{[3]}$. Designers and control engineers have to understand the basic limitations that stem from the process instability ${ }^{[4,5]}$. As real experiments with unstable systems can be hazardous, modelling and simulation tools play important roles in designing safe control systems for such processes nowadays. These tools enable safe experiments, analysis of dangerous states of the systems, design of convenient control, and much more.

The presented site devoted to unstable systems has been developed to enable students, teachers, scientists, and many others interested in unstable processes to have an easy access to the mathematical models of such systems. All these people can easily use the presented models for their own simulation experiments, testing control algorithms and so on. This can lead to a better awareness about unstable processes and the problems they cause. Because the database is easily accessible via the internet ${ }^{[6]}$, it can be used by a wide range of users for various purposes, for example, pedagogical, scientific and others.

This paper is divided into the following sections: After a brief introduction into the stability of dynamic systems, the contribution continues by examples of unstable processes from the site, including their simplified mathematical models together with a brief discussion on their behavior and

\footnotetext{
Regular paper

Special Issue on Recent Advances on Complex Systems Control, Modelling and Prediction II

Manuscript received August 5, 2013; accepted October 11, 2013
}

properties. Further, a basic structure of the developed site is outlined and explained, and the paper concludes by suggesting further possible development of the project. A case study using the database for control system analysis and design is also included at the end of the contribution. Here, the problem of designing a suitable control system for the AMIRA inverted pendulum is addressed and successfully solved using the polynomial approach and some useful tools from the robust control theory.

\section{Stability of dynamic systems}

Stability is the fundamental property of control systems. Therefore, a great deal of effort has been focussed towards the proper definition and testing of system stability and the feasible stabilisation of unstable processes.

\subsection{Defining stability}

Although all people naturally understand the concept of stability and are able to describe what stable behavior is and what is not, a proper mathematical definition is not so straightforward. Generally, stability can be formulated as the ability to recover from perturbations - short-time disturbances or nonzero initial conditions. One of the definitions states that a system is stable if the bounded input into the system produces a bounded output from the system. This is called bounded input-bounded output (BIBO) stability, e.g., $[7,8]$. Another recognised and more general definition is the Lyapunov stability, e.g., [7-10]. Simply speaking, it states that a system is stable if its output and all states are bounded and converge asymptotically to zero from sufficiently small initial conditions.

\section{$2.2 \quad$ Stability testing}

During the past decades, many approaches to stability testing have been developed. The use of a particular method depends on the properties of the system to be tested, for example, if it is linear or nonlinear, continuoustime or discrete-time, time-variant or time-invariant, and so on. The methods can be both numerical and graphical. Interested readers can find details in classical books focussed 
on systems theory or control engineering, e.g., [7, 8, 10,11].

\subsection{Stabilisation}

Unstable systems can be stabilised by feedback. There are many sources focussed on the control system design for unstable processes, e.g., [1,2,12-17]. Many of these works solve the control system design problem connected also with delayed and non-minimum-phase systems, which are also problematic to control.

Besides stability testing and stabilisation of unstable processes, it is often important to test and ensure certain measures of stability, that is, relative stability that gives answer to the question "How far is the system from the instability". For control system design, the so-called gain and phase margins are frequently used; for details, see [8, 10, 11].

The so-called robust stability is the next important term in control engineering. It is used in case we need to test/achieve stability not only for one system but for a certain class of systems, typically a nominal system and some of its neighbourhood, which is useful in the case of uncertain models. Interested readers are referred to classical books devoted to the robust system design, e.g., $[18,19]$.

\section{Examples of unstable systems}

As explained in Section 1, unstable processes are common in many areas of our daily lives. Several such systems are briefly discussed in this section. Presented mathematical models together with simulation files and original sources are also available online from the developed site ${ }^{[6]}$.

\subsection{Nonideal continuous stirred-tank reac- tor}

This process is represented by a continuous stirredtank reactor (CSTR) with nonideal mixing described by Cholette's model. The process can be illustrated according to Fig. 1.

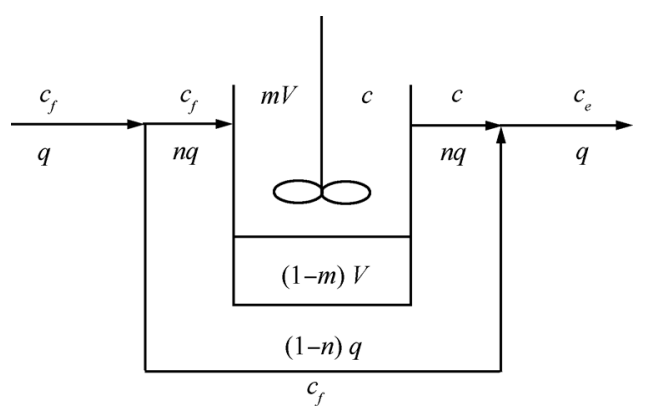

Fig. 1 Nonideal CSTR

A simplified mathematical model of the process dynamics can be described by the following nonlinear differential formulas ${ }^{[2,20]}$ :

$$
\begin{gathered}
\frac{\mathrm{d} c(t)}{\mathrm{d} t}=\frac{n q}{m V}\left[c_{f}(t)-c(t)\right]-\frac{k_{1} c(t)}{\left[1+k_{2} c(t)\right]^{2}} \\
n c(t)+(1-n) c_{f}(t)=c_{e}(t) .
\end{gathered}
$$

In Fig. 1 and (1), $c(t)$ is the concentration of the reactant in the well-mixed zone, $c_{e}(t)$ is the concentration in the exit stream (controlled variable), and $c_{f}(t)$ is the feed concentration (manipulated variable). Further, $n$ is the fraction of the reactant feed that enters the zone of perfect mixing, and $m$ is the fraction of the total volume $V$ of the reactor where the reaction occurs. Constants $k_{1}$ and $k_{2}$ describe the reaction rate, and $q$ is the inlet flow rate. The parameters of the presented model are defined as follows: $n=m=$ $0.75, V=1 \mathrm{l}, q=0.033 \mathrm{l} / \mathrm{s}, k_{1}=10 \mathrm{~s}^{-1}$, and $k_{2}=10 \mathrm{l} / \mathrm{mol}$. And a nominal operating point is described as follows: $c_{f}$ $=6.484 \mathrm{~mol} / 1, c_{e}=1.8 \mathrm{~mol} / 1$, and $c=0.239 \mathrm{~mol} / 1$. The linearisation of the nonlinear model (1) around the given nominal operating point gives the transfer function model as

$$
\frac{\Delta c_{e}(s)}{\Delta c_{f}(s)}=\frac{0.7725 s-0.1727}{3.1 s-1}
$$

A state-space representation of the linearised model in the form:

$$
x^{\prime}(t)=A x(t)+B u(t), \quad y(t)=C x(t)+D u(t)
$$

where $x(t)$ defines a vector of state variables, $y(t)$ defines a vector of output variables, and $u(t)$ defines a vector of input variables, can be obtained as (using the Matlab function "ssdata"):

$$
\begin{gathered}
A=[0.3226], B=[0.1250] \\
C=[0.1974], D=[0.2492] .
\end{gathered}
$$

In the case of the presented reactor, the variables $x(t), y(t)$, and $u(t)$ are only scalars and correspond to the reactor variables $c(t), c_{e}(t)$, and $c_{f}(t)$, respectively. (Consequently, the matrices $A, B, C$, and $D$ are also only scalars.) From the control theory point of view, the models (2)-(4) represent a first-order proper system that is unstable (one positive pole, i.e., denominator root, located at $p_{1}$ $=0.3226$ ) with non-minimum-phase behavior (one positive zero, i.e., numerator root, located at $z_{1}=0.2236$ ) and with gain $k=0.1727$. Such systems, which are both unstable and non-minimum phase, are not so easy to control. The step response of the model recorded in Fig. 2 clearly demonstrates the instability of the system.

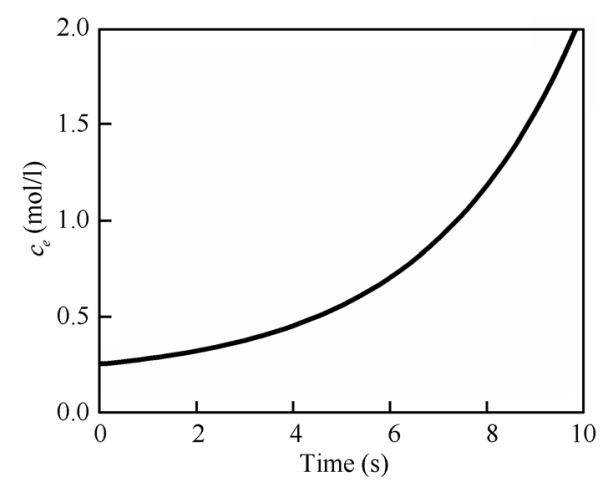

Fig. 2 Nonideal CSTR step response

\subsection{Ballistic missile}

A ballistic missile can represent another unstable system from the military industry. Although it is completely different from the previous one, it shares the property of instability and consequently problematic control. When controlling 
the altitude of the ballistic missile, the transfer function relating the altitude $y(t)$ to the thrust chamber deflection $\delta(t)$ has the following form ${ }^{[2,21]}$ :

$$
\frac{\Delta y(s)}{\Delta \delta(s)}=\frac{7.21(s+0.0526)}{(s+1.6)(s-1.48)(s-0.023)} .
$$

Its state-space representation in the form (3) can be obtained, e.g.,

$$
\begin{aligned}
& A=\left[\begin{array}{ccc}
-0.097 & 1.185 & -0.055 \\
2 & 0 & 0 \\
0 & 0.5 & 0
\end{array}\right], B=\left[\begin{array}{l}
2 \\
0 \\
0
\end{array}\right] \\
& C=\left[\begin{array}{lll}
0 & 1.8025 & 0.1896
\end{array}\right], D=[0] .
\end{aligned}
$$

From the control theory point of view, the missile represents a strictly proper unstable system of the third order. The instability is given by the two poles located in the right half of the complex plane $\left(p_{1}=0.023, p_{2}=1.48\right)$, as illustrated in Fig. 3. The system also has relatively fast dynamics, with time constants in seconds.

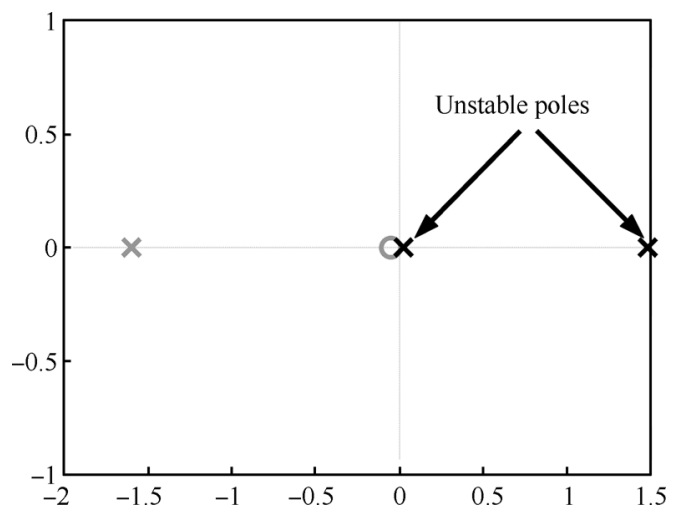

Fig. 3 Pole-zero map of the ballistic missile

\section{$3.3 \quad \mathrm{X}-29$ aircraft}

The X-29 illustrated in Fig. 4 was an experimental aircraft that tested forward-swept wing, canard control surfaces, and other novel aircraft technologies. It was deliberately designed with static instability to increase its manoeuverability and speeds of command response. However, it was impossible to pilot this airplane conventionally by manual flight controls, and it required the use of the socalled fly-by-wire (computerised) control system. In addition, special hardware (sensors, control processors, and actuators) had to be used to stabilise the system over all flight regimes and all loading conditions. Considerable effort has been devoted to the design of the flight control system for this airplane, e.g., [3, 22, 23].

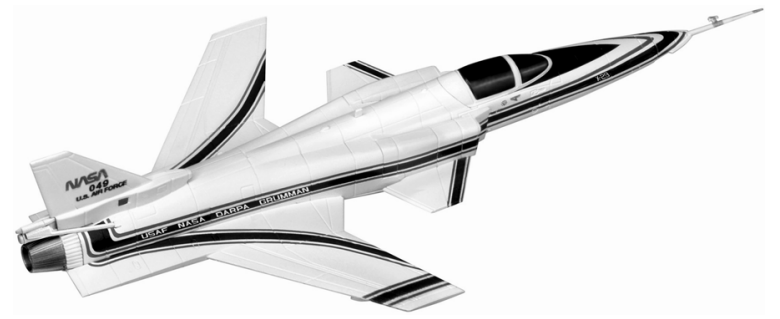

Fig. 4 X-29 aircraft
The benefits of instability (better manoeuverability and faster reaction) were desired in the transonic and supersonic flight regimes, so the airplane was designed to be modestly unstable in those regimes. However, because of a basic aerodynamic phenomenon, the X-29's slight instability at supersonic speeds turned into a much more dramatic instability at subsonic speeds. A simplified linearised model at one such flight condition given by a transfer function has the following form:

$$
G(s)=\frac{s-26}{s-6}
$$

As can be clearly seen, the airplane's real pole (denominator root) is as large as $+6 \mathrm{rad} / \mathrm{s}$, which makes this system nearly impossible to control manually. It can be compared, simply speaking, to balancing a 1-ft-long stick ${ }^{[3]}$. Besides the unstable pole, the system has also a strong non-minimum-phase behavior, that is, inverse response (a zero-numerator root, located at $z_{1}=26$ ). These facts make this system very difficult to control. The state-space realisation of the model (7) in the form (3) can be obtained, for example, as

$$
A=[6], B=[4], C=[-5], D=[1] .
$$

Next, Fig. 5 shows the sensitivity function $S(\omega)$ of a X-29 prototype.

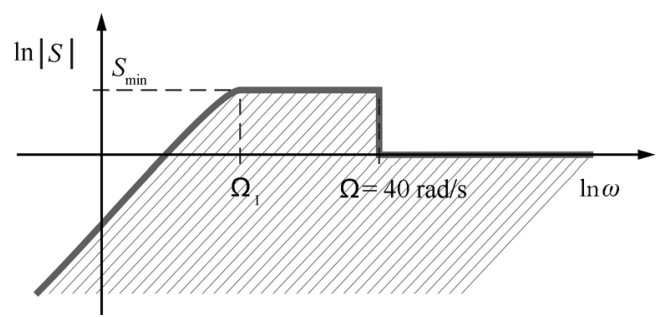

Fig. 5 X-29 sensitivity function

From the plot, it can be seen that the system has considerably limited bandwidth (up to $40 \mathrm{rad} / \mathrm{s}$ ), which is given by the used hardware components (sensors, control processors, and actuators), airplane mechanical structure, and aerodynamics conditions. Consequently, it narrows the possibilities of convenient control system design.

\subsection{Inverted pendulum}

An inverted pendulum is a classic example of an unstable system. The AMIRA implementation ${ }^{[24]}$ shown in Fig. 6 consists of a cart (denoted 6 in the figure), which can be moved along a metal guiding bar (5). An aluminium rod (9) with a cylindrical weight is fixed to the cart by an axis.

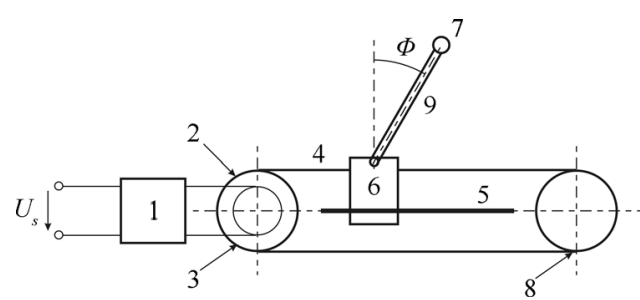

Fig. 6 Inverted pendulum AMIRA 
This system has one input and two outputs. Input signal is the control voltage of a DC motor (2), which can change the position of the cart. The outputs are cart position and angle of the pendulum rod. Both outputs are measured by incremental encoders. The basic control task is to hold the pendulum rod in the upper upright position. The mathematical model of the system dynamics can be described by the following pair of nonlinear differential equations ${ }^{[24-26]}$ :

$$
\begin{gathered}
m \ddot{r}+F_{\mathrm{r}} \dot{r}+m_{P} l \ddot{\varphi} \cos \varphi-m_{P} l(\dot{\varphi})^{2} \sin \varphi=F \\
\Theta \ddot{\varphi}+C \dot{\varphi}-m_{P} l g \sin \varphi+m_{P} l \ddot{r} \cos \varphi=0
\end{gathered}
$$

where $F$ represents input signal, which is the force produced by the DC motor. The output signals are $r$ and $\varphi$, which are the cart position ( $\dot{r}$ denotes cart speed) and the pendulum angle ( $\dot{\varphi}$ denotes pendulum angular speed), respectively. The symbol $g$ is the gravity acceleration constant, and $F_{r}$ represents constant of a velocity proportional friction of the cart. The following substitutions were used in (9) and (10):

$$
\begin{aligned}
& \Theta=\Theta_{S}+m_{P} l^{2} \\
& m=m_{C}+m_{P}
\end{aligned}
$$

where $m_{C}$ is the cart weight, $m_{P}$ is the pendulum weight, $l$ is the distance between the centre of gravity of the pendulum and the centre of rotation of the pendulum, and $\Theta_{S}$ represents the inertia moment of the pendulum rod with respect to the centre of gravity. All the used constants and symbols are clearly defined in Table 1 , where the constant $k_{A}$ defines the gain of the servo amplifier (denoted 1 in Fig. 6).

Table 1 Inverted pendulum parameters

\begin{tabular}{ccc}
\hline Parameter & Symbol & Value and unit \\
\hline Cart weight & $m_{C}$ & $4.0 \mathrm{~kg}$ \\
Pendulum weight & $m_{P}$ & $0.36 \mathrm{~kg}$ \\
Total weight & $m$ & $4.36 \mathrm{~kg}$ \\
Pendulum length & $l$ & $0.420 \mathrm{~m}$ \\
Inertia moment & $\Theta$ & $0.08433 \mathrm{~kg} \cdot \mathrm{m}^{2}$ \\
Cart friction & $F_{r}$ & $6.5 \mathrm{~kg} / \mathrm{s}$ \\
Pendulum friction & $C$ & $0.00652 \mathrm{~kg} \cdot \mathrm{m}^{2} / \mathrm{s}$ \\
Rate constant & $k_{A}$ & $7.50 \mathrm{~N} / \mathrm{V}$ \\
\hline
\end{tabular}

The linearisation of the pendulum dynamics $((9)$ and (10)) in the top unstable position of the pendulum rod $(\varphi$ $=0$ ) gives the following transfer function of the system relating the pendulum rod angle with respect to the control voltage of the DC motor:

$$
\begin{aligned}
G(s)= & \frac{-0.4385 s}{s^{3}+1.672 s^{2}-18.63 s-27.96}= \\
& \frac{-0.4385 s}{(s-4.25)(s+1.48)(s+4.45)} .
\end{aligned}
$$

From the control theory point of view, the transfer function describes a derivative system (zero, i.e., numerator root located at $z_{1}=0$ ) with unstable behavior (one positive pole, i.e., numerator root located at $p_{1}=4.25$ ). One possible state-space realisation is given as

$$
\begin{aligned}
& A=\left[\begin{array}{ccc}
-1.67 & 4.66 & 3.50 \\
4.00 & 0 & 0 \\
0 & 2.00 & 0
\end{array}\right], B=\left[\begin{array}{c}
0.25 \\
0 \\
0
\end{array}\right] \\
& C=\left[\begin{array}{lll}
0 & -0.4385 & 0
\end{array}\right], D=[0] .
\end{aligned}
$$

All the models discussed here are available online at the presented site ${ }^{[6]}$, with short description, downloadable model in the Matlab/Simulink environment, and selected references related to modelling, simulation, control system analysis and design of the systems. The next section briefly presents the main structure of the developed site devoted to unstable systems and outlines the main possibilities it offers for prospective users.

\section{Site structure and choices}

The database of unstable systems has been developed as an open, easily extensible system in a bilingual version (English/Czech). It is accessible online at http://www.unstable-systems.cz. A starting version of the site was implemented within the final work [27] using a free and open source content management system Joomla (version 2.5, available online at http://www.joomla.org and described in detail in, e.g., [28]). This makes the administration of the site very easy. It uses the open source database MySQL for storing necessary files and data. The basic structure of the web presentation is divided into several bookmarks: home, unstable systems, stability, contact us, and about project. Apart from this basic structure, there are also user-related services such as registration, profile editing, and web administration.

The Home bookmark is the starting point of the site. It introduces the purpose of the site, enables registration, and shows the latest news and recent posts together with simple statistics and some useful links. Registered users have the following possibilities:

1) Access to files with the simulation models of the systems.

2) Access to latest news via a newsletter.

3) Possibility of articles rating.

4) Possibility to add comments to the systems models.

It is also possible to search within the site and change language (English/Czech).

The Unstable Systems bookmark is the main part of the site that contains the mathematical models of unstable processes. So far (October 2013), it contains following models:

1) Ballistic missile.

2) Fluidised bed reactor.

3) Inverted pendulum.

4) Magnetic levitation system.

5) Nonideal CSTR.

6) X-29 aircraft.

Every model has the following information:

1) Brief description of the system.

2) Scheme of the process or picture of the system.

3) Simplified mathematical model.

4) Definition of used variables and parameters.

5) Downloadable model in, e.g., Matlab/Simulink. 
6) Sources of further information.

It is also possible to generate a printable version of the models or send a link of a model by e-mail. Registered users can download the simulation models, add comments, and rate them. Besides this, it is also possible to search within the models and sort them according to various criteria.

The presented models are being obtained from available sources - books, journal articles, and proceedings papers on the subject and freely accessible online databases.

In order to avoid problems with the different versions of simulation files in, e.g., the Matlab environment, all simulation models are implemented in older versions, which are usually usable in newer versions without problems. Besides this, all simulation files have the version information included to avoid possible difficulties.

The Stability bookmark explains the basics about the stability of systems - general understanding of the term, several definitions, such as BIBO, and the Lyapunov stability. It also offers further reading on this subject.

The next bookmark, contact us, enables to send questions, suggestions and remarks, etc. on the site and models to the authors. The final bookmark, About Project, briefly introduces basic information about this project, including main authors, brief description, and terms of use.

The next section briefly illustrates the potential of the site when designing a control system for one of the presented systems - the inverted pendulum.

\section{Case study of using the database}

Suppose a control designer is wondering if his/her method is also suitable for control of unstable systems. He chooses the system of inverted pendulum from the database for testing. Besides basic information about the system, he gains the mathematical model of the system with all its parameters together with a simulation file in Matlab/Simulink for simulation experiments and testing. Both nonlinear (time domain) and linear (complex domain) versions of the model enable the designer to choose an appropriate method for control system analysis and design. Suggested further reading and source of the presented information can also help with this task. Further in this section, one such example of control system analysis and design follows.

Assume the linearised version of the inverted pendulum model in the form of the continuous-time transfer function in the complex Laplace variable $s$ in (13) is

$$
G(s)=\frac{-0.4385 s}{s^{3}+1.672 s^{2}-18.63 s-27.96}=\frac{b(s)}{a(s)} .
$$

This model is valid only in the neighbourhood of the top upright (unstable) position of the pendulum rod and relates the pendulum angle to the voltage of the driving motor. As the model is described by rational function in polynomials $b(s)$ and $a(s)$, the control design method will follow the polynomial approach, which is a systematic methodology enabling to find both suitable structure and parameters of a controller. The basics of this approach can be found in, e.g., [29-31]. Suppose the classical control setup of Fig. 7, where $G$ denotes a plant to be controlled by a controller $C$ and the signals $w, e, u$, and $y$ stand for the reference (set point), control error, control input (manipulated variable), and a process (controlled) variable, respectively. Signals $v_{u}$ and $v_{y}$ represent general disturbances.

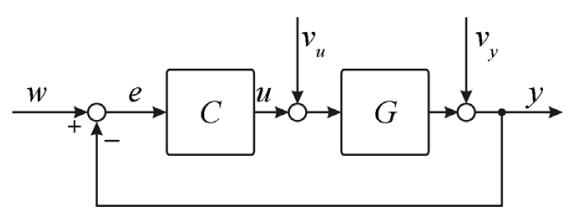

Fig. 7 Control system configuration

Let us assume that the controller $C$ can be also described by a transfer function (16) with $q(s)$ and $p(s)$ coprime polynomials satisfying (17), that is, the controller is generally assumed to be proper.

$$
\begin{aligned}
C(s) & =\frac{q(s)}{p(s)} \\
\operatorname{deg} p(s) & \geqslant \operatorname{deg} q(s) .
\end{aligned}
$$

The basic requirements for the control system introduced above are formulated as follows:

1) Stability.

2) Asymptotic tracking of the reference signal.

3) Disturbances attenuation.

4) Inner properness.

Besides the above-mentioned general requirements, the control system should also be robust to cope with the real nonlinear system (not only with the adopted linear model) and possible disturbances. This is especially important in this case when dealing with unstable systems.

From the scheme of Fig. 7 and assuming (16) and (15), it is easy to derive following relationships between the controlled variable $y$ ( $Y$ in the complex domain) and the input signals $w, v_{u}$, and $v_{y}\left(W, V_{u}\right.$, and $V_{y}$ similarly). The argument $s$ is omitted in these formulas to keep them more compact and readable):

$$
\begin{aligned}
Y= & \frac{G C}{1+G C} W+\frac{G}{1+G C} V_{u}+\frac{1}{1+G C} V_{y}= \\
& \frac{b q}{a p+b q} W+\frac{b p}{a p+b q} V_{u}+\frac{a p}{a p+b q} V_{y}= \\
& \frac{b q}{d} W+\frac{b p}{d} V_{u}+\frac{a p}{d} V_{y}= \\
& T W+S_{u} V_{u}+S V_{y} .
\end{aligned}
$$

Here, $d$ defines a characteristic polynomial of the closed loop, and consequently, it is given as

$$
a p+b q=d .
$$

Symbols $S, T$, and $S_{u}$ denote important transfer functions of the loop known as the sensitivity function, the complementary sensitivity function, and the input sensitivity function, respectively. The sensitivity function $S$ is further used to make the designed control system robust.

Similarly, it is straightforward to derive (20) for the control error $e(E(s)$ in the complex domain):

$$
E(s)=\frac{p}{d}\left[a W(s)-b V_{u}(s)-a V_{y}(s)\right] .
$$


1) Control system stability.

From (18), it is clear that the control system of Fig. 7 . will be stable if the characteristic polynomial $d(s)$ given by (19) is stable. This Diophantine equation, after a proper choice of the stable polynomial $d(s)$, is used to compute unknown controller polynomials $q(s)$ and $p(s)$.

2) Asymptotic tracking of the reference signal and disturbance attenuation.

Let us assume that the reference signal $w(t)$ is a step function defined in the complex domain as

$$
W(s)=\frac{w_{0}}{s}
$$

and further, suppose that both disturbances $v_{u}(t)$ and $v_{y}(t)$ can be also approximated by the following step functions:

$$
V_{u}(s)=\frac{v_{u 0}}{s}, \quad V_{y}(s)=\frac{v_{y 0}}{s} .
$$

Then, substituting (21) and (22) into (20) yields

$$
E(s)=\frac{p}{d}\left(a \frac{w_{0}}{s}-b \frac{v_{u 0}}{s}-a \frac{v_{y 0}}{s}\right)
$$

which shows that to guarantee zero-control error in the steady state (despite both disturbances), the denominator polynomial of the controller $p(s)$ needs to be divisible by the $s$ term. Further inspection of the formula for the case of the derivative system of the inverted pendulum (15) shows that the controller has to include double integrator, which will be fulfilled for its denominator polynomial in the following form:

$$
p(s)=s^{2} \tilde{p}(s) .
$$

Then the controller (16) can be written as

$$
C(s)=\frac{q(s)}{s^{2} \tilde{p}(s)}
$$

and the Diophantine equation (19) defining stability will be

$$
a s^{2} \tilde{p}+b q=d .
$$

3) Inner properness of the control system.

The inner properness of the control system is satisfied if all its parts (transfer functions) are proper. With regard to the strictly proper transfer function $G(s)$ (15), proper controller (16) and (17), and taking into account the solvability of (19) and (26), it is possible to derive the following equations for the degrees of the unknown polynomials $q, \tilde{p}$, and $d$ :

$$
\begin{aligned}
& \operatorname{deg}\{q(s)\}=\operatorname{deg}\{a(s)\} \\
& \operatorname{deg}\{\tilde{p}(s)\} \geqslant \operatorname{deg}\{a(s)\}-2 \\
& \operatorname{deg}\{d(s)\} \geqslant 2 \times \operatorname{deg}\{a(s)\}-1 .
\end{aligned}
$$

Then, the resultant controller takes the general form

$$
Q(s)=\frac{q_{3} s^{3}+q_{2} s^{2}+q_{1} s+q_{0}}{s^{2}\left(\tilde{p}_{1} s+\tilde{p}_{0}\right)} .
$$

In order to compute the coefficients of this controller from (19) and (26), the stable polynomial $d(s)$ must be determined. Here, it is suggested to have it in this form:

$$
d(s)=n(s)(s+\alpha)^{2}
$$

where $\alpha \succ 0$ is a tuning constant and $n(s)$ is a stable polynomial computed from the polynomial of the controlled system $a(s)$ using the following spectral factorisation technique ${ }^{[32]}$ :

$$
a^{*}(s) a(s)=n^{*}(s) n(s) .
$$

The asterisk denotes a complex conjugate polynomial, $x^{*}(s)=x(-s)$, and the result of the factorisation is a polynomial with the similar properties as the original, but stable.

This choice of the characteristic polynomial will guarantee not only the stability of the resultant control system but also the connection to the original process behavior, and it will leave space enough for further possible tuning as well.

4) Robust setting of the designed loop.

In order to cope with external disturbances and with the fact that only an approximate model of a nonlinear unstable plant is used for the control system design, the closed loop is designed to be robust. This is done with the help of the sensitivity function $S$ from (18). The sensitivity function $S$ is defined as

$$
\begin{aligned}
S(s)= & \frac{Y(s)}{V_{y}(s)}=\frac{1}{1+G(s) C(s)}= \\
& \frac{a(s) p(s)}{a(s) p(s)+b(s) q(s)}=\frac{a(s) p(s)}{d(s)}
\end{aligned}
$$

and it describes the impact of output disturbance $v_{y}$ on the process output $y$. Moreover, it gives the relative sensitivity of the closed-loop transfer function $T(s)$ to the relative plant model error. The peak gain of its frequency response given by the infinity norm $H_{\infty}$ is a good measure of the loop robustness, e.g., [8]. Therefore, in this work, it is suggested to use the sensitivity function and its $H_{\infty}$ norm to determine the free tuning parameter $\alpha \succ 0$ from the closed-loop characteristic polynomial (29) to make the designed control system more robust, that is, safer.

The dependence of the $H_{\infty}$ norm of the sensitivity function (31) upon the tuning parameter $\alpha$ is depicted in Fig. 8.

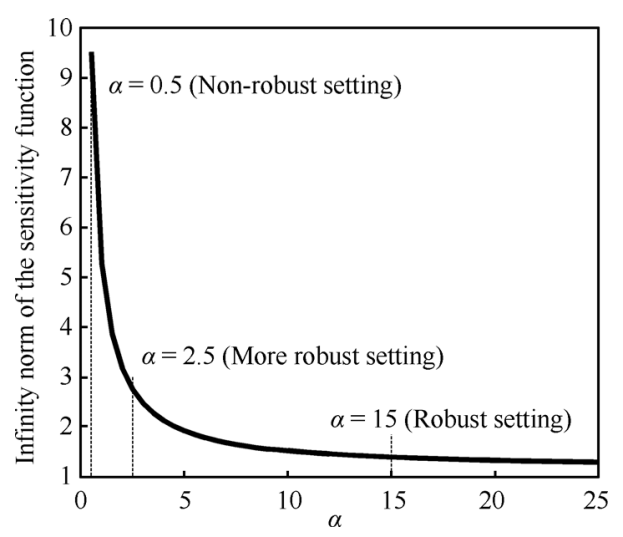

Fig. 8 Infinity norm of the sensitivity function $S$ with $\alpha$

Fig. 8 shows that the smaller the value of parameter $\alpha$ is, the more sensitive the closed-loop system is; and the higher value of $\alpha$ is, the more robust the control system is (regarding the influence of disturbances and possible perturbations in the process model). Selected choices of $\alpha=$ 
$\{0.5,2.5,15\}$ (highlighted in Figs. 8 and 9) show that the first choice $(\alpha=0.5)$ represents a non-robust setting with high sensitivity to disturbances and modelling errors, which is also apparent from Fig. 9, where control responses for the selected values of $\alpha$ are presented (here, during the control process, the step disturbance of the amplitude $-0.07 \mathrm{rad}$ influenced the controlled output in the time $t \in(15 ; 15.5) \mathrm{s}$ to test the sensitivity of the designed loop to disturbances).
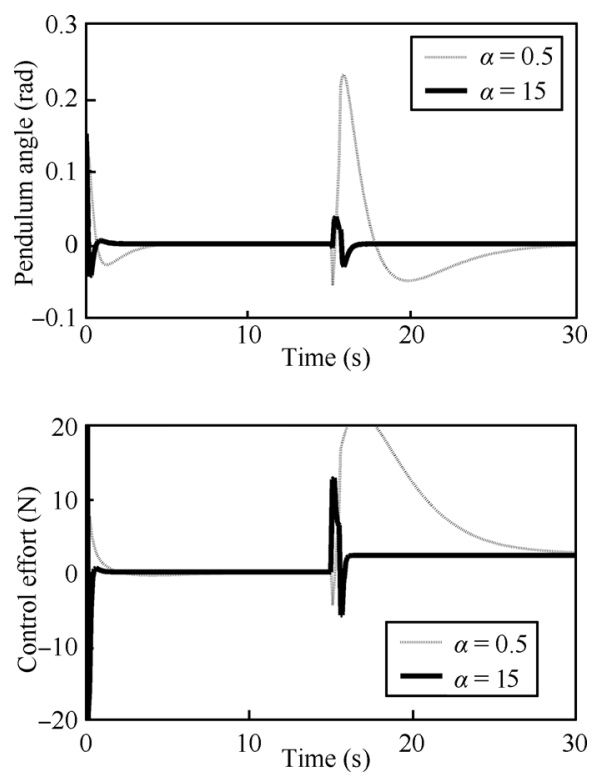

Fig. 9 Inverted pendulum control response

The most robust setting is the third one with $\alpha=15$, where the disturbance is attenuated very quickly but with greater control effort. Therefore, it is always advisable to seek a reasonable trade-off between the robustness of the designed loop and the practical limitation on the control input and its changes, especially when dealing with unstable processes.

The resulting controllers for the different settings of the tuning parameter $\alpha$ are presented in Table 2 .

Table 2 Designed controllers for different values of $\alpha$

\begin{tabular}{cc}
\hline Tuning parameter $\alpha$ & Controller $C(s)$ \\
\hline 0.5 & $\frac{-102.5 s^{3}-609.7 s^{2}-688.1 s-15.9}{s^{2}(s+9.5)}$ \\
2.5 & $\frac{-193.8 s^{3}-1209 s^{2}-1633 s-398.5}{s^{2}(s+13.5)}$ \\
15 & $\frac{-1178 s^{3}-9161 s^{2}-20670 s-14350}{s^{2}(s+38.5)}$ \\
\hline
\end{tabular}

This brief study has shown how the information and simulation models from the developed database of unstable systems can be utilised, e.g., for control system analysis and design.

\section{Conclusions}

Modelling and simulation tools play important roles in our lives nowadays. In the case of unstable systems anal- ysis and control system design, their role is crucial. Experiments with such systems without the proper knowledge about possible consequences can be very hazardous ${ }^{[3]}$. The goal of this contribution was to present the starting project of the web-based database of unstable systems. The site can help students, teachers, designers, scientists, and many others to understand the basic properties of unstable systems. This is done via available models of the systems/processes and suggested further readings. The developed web site is an open, constantly developing system. Therefore, the experiences of users and their suggestions, remarks, and comments are welcome.

Further development of the site will be focussed on the extension of the given information concerning the systems stability, description of the models, and suggested further readings. The number of available unstable systems models will grow as well as the number of simulation files (not necessarily limited to the Matlab/Simulink environment). Another attractive extension can be seen in the possibility to implement simulation directly into the site, for example, using the popular Easy Java Simulations open-source software tool ${ }^{[33]}$. At the moment, only the site administrator can add articles and models into the system. The possibility to do this by registered users is also being considered, which would certainly help to further develop the database. Besides the simulation models of the unstable systems, also simulation files related to control system design for such processes can be added gradually.

\section{Acknowledgement}

The author thanks his students, Jaroslav Kolařík and Jiří Marholt, for helping in the development of the starting version of the database and corresponding simulation files.

\section{References}

[1] M. Chidambaram. Control of unstable systems: A review. Journal of Energy, Heat and Mass Transfer, vol. 19, pp. 4957, 1997.

[2] R. Padma Sree, M. Chidambaram. Control of Unstable Systems, Oxford: Alpha Science Int. Ltd., 2006.

[3] G. Stein. Respect the unstable. IEEE Control System Magazine, vol. 23 , no. 4, pp. 12-25, 2003.

[4] R. H. Middleton. Trade-offs in linear control system design. Automatica, vol. 27, no. 2, pp. 281-292, 1991.

[5] S. Skogestad, K. Havre, T. Larsson. Control limitations for unstable plants. In Proceedings of the 15th Triennial World Congress, IFAC, Barcelona, Spain, pp. 328, 2002.

[6] F. Gazdoš, J. Kolařík. Database of Unstable Systems, [Online], Available: http://www.unstable-systems.cz, October $22,2013$.

[7] J. L. Willems. Stability Theory of Dynamical Systems, New York: Wiley, 1970.

[8] S. Skogestad, I. Postlethwaite. Multivariable Feedback Control: Analysis and Design, Chichester: Wiley, 2005.

[9] P. C. Parks. A. M. Lyapunov's stability theory-100 years on. IMA Journal of Mathematical Control and Information, vol. 9, no. 4, pp. 275-303, 1992.

[10] K. J. Åström, R. M. Murray. Feedback Systems: An Introduction for Scientist and Engineers, Princeton: Princeton University Press, 2008.

[11] J. C. Doyle, B. A. Francis, A. R. Tannenbaum. Feedback Control Theory, Mineola, New York: Dover Publications, 2009 . 
[12] J. H. Park, S. W. Sung, I. B. Lee. An enhanced PID control strategy for unstable processes. Automatica, vol. 34, no. 6 , pp. 751-756, 1998.

[13] G. Marchetti, C. Scali, D. R. Lewin. Identification and control of open-loop unstable processes by relay methods. $\mathrm{Au}$ tomatica, vol. 37, no. 12, pp. 2049-2055, 2001.

[14] R. Lozano, P. Castillo, P. Garcia, A. Dzul. Robust prediction-based control for unstable delay systems: Application to the yaw control of a mini-helicopter. Automatica, vol. 40, no. 4, pp. 603-612, 2004.

[15] P. García, P. Albertos, T. Hägglund. Control of unstable non-minimum-phase delayed systems. Journal of Process Control, vol. 16, no. 10, pp. 1099-1111, 2006.

[16] P. Dostál, F. Gazdoš, V. Bobál. Design of controllers for time delay systems, Part II: Integrating and unstable systems. Journal of Electrical Engineering, vol. 59, no. 1, pp. 38, 2008.

[17] X. K. Zhang, X. L. Jia. Solving robust controller of unstable process using mirror-injection method. Systems Engineering and Electronics, vol. 22, no. 4, pp. 10-12, 2000.

[18] B. R. Barmish. New Tools for Robustness of Linear Systems, New York: Macmillan, 1994.

[19] S. P. Bhattacharyya, H. Chapellat, L. H. Keel. Robust Control: The Parametric Approach, New Jersey: Prentice-Hall, 1995.

[20] C. T. Liou, Y. S. Chien. The effect of nonideal mixing on input multiplicity in a CSTR. Chemical Engineering Science, vol. 46, no. 8, pp. 2113-2116, 1991.

[21] J. H. Blakelock. Automatic Control of Aircraft and Missiles, New York: John Wiley, 1991.

[22] W. L. Rogers, D. J. Collins. X-29 $H_{\infty}$ controller synthesis. Journal of Guidance, Control, and Dynamics, vol. 15, no. 4, pp. 962-967, 1992.

[23] R. Clarke, J. J. Burken, J. T. Bosworth, J. E. Bauer. X-29 flight control system: Lessons learned. International Journal of Control, vol. 59, no. 1, pp. 199-219, 1994.

[24] AMIRA. PS600 Laboratory Experiment Inverted Pendulum, Amira GmbH, Duisburg, Germany, 2000.

[25] P. Chalupa, V. Bobál. Modelling and predictive control of inverted pendulum. In Proceedings of the 22nd European Conference on Modelling and Simulation, EDMS, Nicosia, Cyprus, pp. 531-537, 2008.

[26] J. Marholt, F. Gazdoš, P. Dostál. Control of the unstable system of the inverted pendulum using the polynomial approach. Cybernetic Letters, 2011.
[27] J. Kolařík. Web-based Database of Unstable Systems, Bachelor Dissertation, Faculty of Applied Informatics, Tomas Bata University in Zlín, Czech Republic, 2012. (in Czech)

[28] J. Marriott, E. Waring. The Official Joomla! Book, 2nd ed., Boston: Addison-Wesley Professional, 2013.

[29] V. Kučera. Diophantine equations in control-a survey. Automatica, vol. 29, no. 6, pp. 1361-1375, 1993.

[30] K. J. Hunt. Polynomial Methods in Optimal Control and Filtering, London: Peter Peregrinus Ltd., 1993.

[31] B. D. O. Anderson. From Youla-Kucera to identification, adaptive and nonlinear control. Automatica, vol. 34, no. 12 pp. 1485-1506, 1998.

[32] M. J. Grimble. Robust Industrial Control: Optimal Design Approach for Polynomial Systems, London: Prentice Hall, 1994.

[33] F. Esquembre. Easy Java Simulations, [Online], Available: http://fem.um.es/Ejs, October 22, 2013.

[34] F. Gazdoš, J. Marholt, J. Kolařík. Unstable systems database: A new tool for students, teachers and scientists. In Proceedings of Nostradamus 2013: Prediction, Modeling \& Analysis of Complex Systems, Springer, Ostrava, Czech Republic, pp. 275-284, 2013.

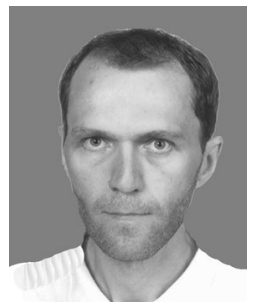

František Gazdoš graduated from the Brno University of Technology, Czech Republic in 1999 with the M.Sc. degree in automation. He then followed studies of technical cybernetics at Tomas Bata University in Zlín, Czech Republic, obtaining his Ph. D. degree in 2004. He became an associate professor in machine and process control in 2012 and now works at the Department of Process Control, Faculty of Applied Informatics, Tomas Bata University in Zlín, Czech Republic. He is an author or a coauthor of more than 70 journal contributions and conference papers giving lectures at foreign universities, such as Politecnico di Milano, University of Strathclyde Glasgow, and Universidade Técnica de Lisboa, among others.

His research interests include modelling, simulation, and control of technological processes.

E-mail: gazdos@fai.utb.cz 